\title{
DAILY MAPPING OF LANDSAT-LIKE LAI AND CORRELATION TO GRAPE YIELD
}

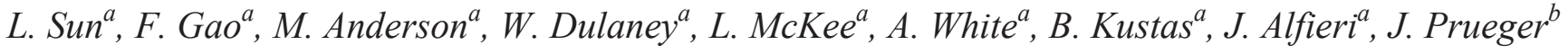 \\ aUSDA-ARS, Hydrology and Remote Sensing Laboratory, Beltsville, MD, USA \\ bUSDA-ARS, National Laboratory for Agriculture and the Environment, Ames, IA, USA
}

\begin{abstract}
Wine grape quality and quantity are affected by vine growing conditions during some critical growing stages. In this paper, MODIS and Landsat were used to map daily LAI in two Grape Remote sensing Atmospheric Profiling and Evapotranspiration eXperiment (GRAPEX) experimental fields near Lodi, California in 2013. A data fusion approach was applied to map daily LAI at $30 \mathrm{~m}$ spatial resolution. The LAI data from two fields were validated using insitu measurements. Cumulative LAI from two fields were compared to the 2013 grape yields. High correlations were found between yield and cumulative LAI. The within-season yield predictability was examined using daily LAI maps. Results show that the grape yields were highly correlated with the cumulative LAI after the month of June. This suggests that grape yields are predictable using remotely sensed data 2-3 months prior to harvest under normal conditions.
\end{abstract}

Index Terms - MODIS, Landsat, LAI, grape, yield

\section{INTRODUCTION}

California, which produces over $90 \%$ of the wine grown in the United States, has seen steady growth in the amount of acreage dedicated to wine-grape production with approximately 615,000 acres planted in 2014 , up from 610,000 in 2013 . For viticultural decision making, information on growing conditions is most useful at the field or sub-field scales, making the $30 \mathrm{~m}$ resolution of Landsat imagery particularly important. Unfortunately, Landsat overpass frequency is often insufficient. While other satellites provide daily coverage (e.g., geostationary satellites, and moderate resolution polar orbiting systems like the Moderate Resolution Imaging Spectroradiometer [MODIS]), these sensors are too coarse in resolution to provide the required field-scale information. In this study we provide an integrated multi-sensor approach that combines the benefits of high spatial resolution Landsat imagery with high temporal resolution MODIS data. This approach provides daily field-scale growing condition estimates that are of significant benefit to vineyard managers.

\section{METHOD}

\subsection{Landsat-like LAI estimation}

Leaf area index (LAI) is a key parameter in most land surface models. Models that operate at multiple spatial scales require consistent LAI inputs at different 
spatial resolutions or from different sensors. In order to make a LAI product consistent with MODIS data from Landsat imagery, Gao et al. (2012) used homogeneous and high quality LAI retrievals from MODIS scenes as references in order to develop a regression tree which related MODIS LAI samples to Landsat surface reflectances [1].

2.2 Daily LAI interpolated method

Although NASA's MODIS biophysical products provide high quality biophysical parameters, the MODIS time series occasionally can contain lower quality data, gaps from persistent clouds, cloud contamination and other missing data. A phenological time series computer program, TIMESAT[2], was used to smooth the MODIS LAI curves with a local polynomial function classified as an adaptive Savitzky-Golay filter. The smoothed LAI curve is used for generating daily LAI maps and extracting seasonal parameters related to the growing season.

3. Results

3.1 Validation by ground LAI.

We compared the estimated Landsat LAI with the field measured LAI in 2013. The grape LAI was measured at the north and south sites (figure 1). The fieldmeasured LAI from the LI-COR LAI-2200 plant canopy analyzer is consistent with Landsat LAI estimates.
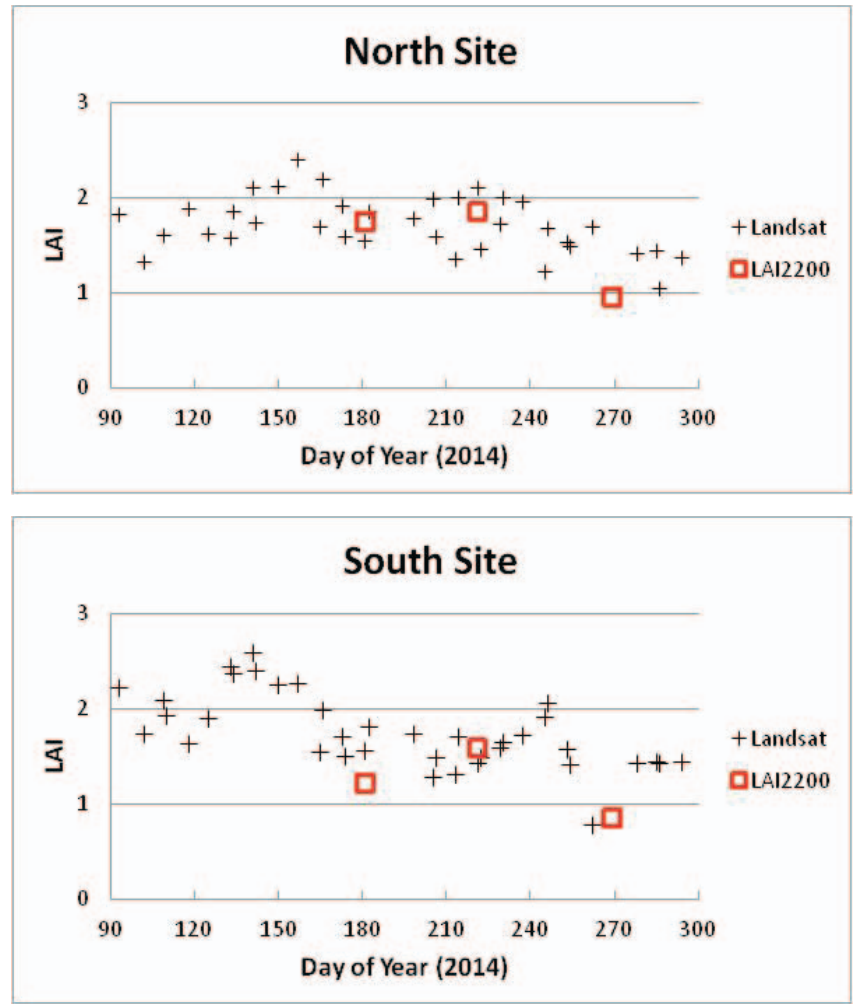

Figure 1 Comparison of Landsat LAI and field measured LAI

\subsection{Landsat-like LAI}

Figure 2 shows the estimated $30 \mathrm{~m}$ resolution LAI distribution in the Gallo experimental field site on DOY 82, 170 and 250. These dates correspond to the start, middle and end of the growing season. The LAI values are relatively low at the start of the season (DOY 82), and reach to almost the highest value at the middle of the season (DOY 170), then became smaller at the end of the season. The high value from the south field on DOY 82 is due to the spectral contribution of the background grass. 


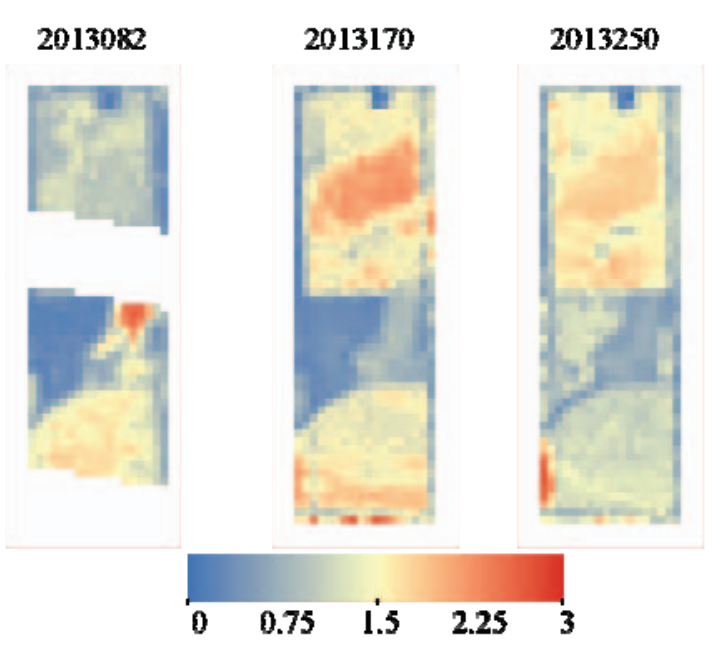

Figure 2 Estimated 2013 Landsat LAI distribution

The distribution of yield, total LAI and maximum LAI during the 2013 growing season is shown in figure 3. These surface parameters have a similar distribution. The middle area of the north field has the highest values. As seen in figure 4, the total LAI has a better correlation with yield $\left(\mathrm{R}^{2}=0.767\right)$ than the maximum LAI $(R 2=0.697)$ which indicates that the total LAI, or cumulative LAI, is a good factor to predict yield. Statistics of the cumulative LAI from April (start of growing season) to September (end of growing season) and the R2 between cumulative LAI from different time periodsand yield are shown in figure 5. The results show that R2 increasedas the growing season came to an end, reaching the highest value in August with September close to the August value.

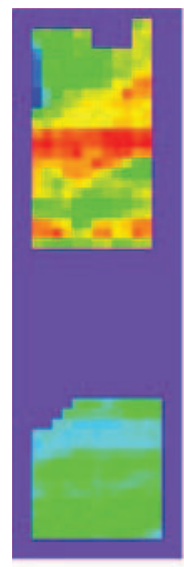

Yield

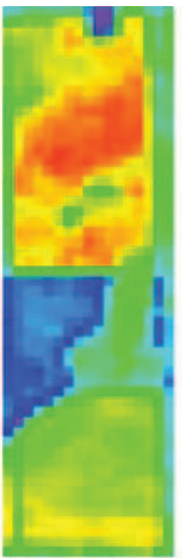

total LAI

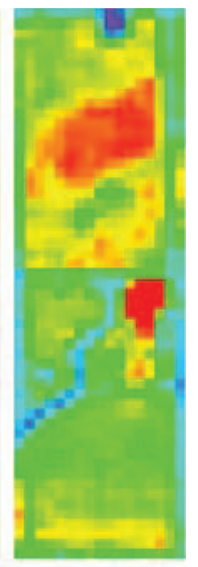

$\max$ LAI
Figure 3 Yield, total LAI and max LAI of 2013
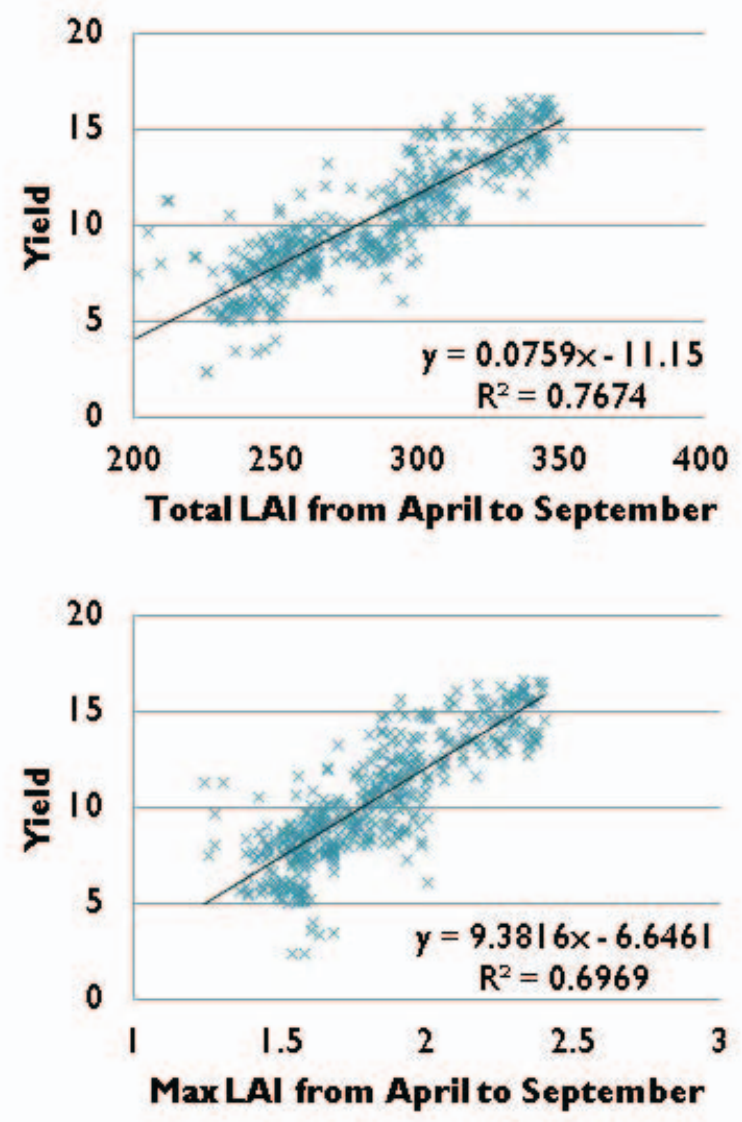

Figure 4. scatter plots between total LAI, maximum LAI and yield 


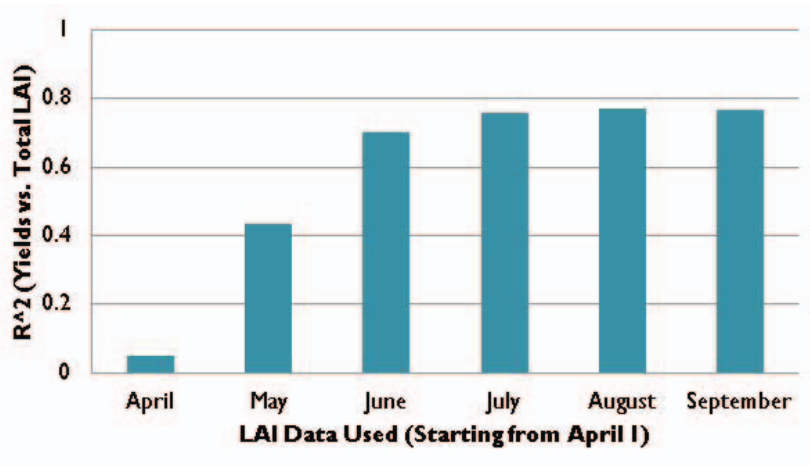

Figure 5 Compute $\mathrm{R}^{2}$ between total LAI during the period and yield

\section{CONCLUSION}

Landsat and Moderate Resolution Imaging Spectroradiometer (MODIS) data products can be used to extract grape vine leaf area index (LAI) and phenology. In this paper, a data fusion approach was applied to map daily LAI at $30 \mathrm{~m}$ spatial resolution. The LAI data from two fields were validated using insitu measurements. The cumulative LAI from two fields were compared to grape yields from two years. High correlations were found between yield and cumulative ET as well as cumulative LAI. The withinseason yield predictability was examined using daily LAI maps. Results show that grape yields were highly correlated with cumulative LAI after the month of June suggesting that grape yields are predictable using remotely sensed data 2-3 months prior to harvest under normal conditions. In the future, a more accurate yield prediction model will be developed based on remotely sensed LAI, soil moisture [4] and evapotranspiration information [5].
[1] Gao, F., Anderson, M.C., Kustas, W.P., Wang, Y. (2012). Simple method for retrieving leaf area index from Landsat using MODIS LAI products as reference. Journal of Applied Remote Sensing, 6, 063554 (2012), doi:10.1117/1.JRS.6.063554.

[2] Jonsson, P. and Eklundh, L. (2004). TIMESAT - a program for analysing time-series of satellite sensor data, Computers and Geosciences 30, 833-845. doi:10.1016/j.cageo.2004.05.006

[3] Bramley, R.G.V., \& Williams, S.K. (2001). A protocol for the construction of yield maps from data collected using commercially available grape yieldmonitors. Adelaide, S.A.: Cooperative Research Centre for Viticulture.

[4] Sun Liang, Sun Rui, Li Xiaowen, Liang Shunlin, Zhang Renhua. Monitoring surface soil moisture status based on remotely sensed surface temperature and vegetation index information, Agriculture and Forest Meteorology, 2012, 166-167: 175-187. doi:10.1016/j.agrformet.2012.07.015

[5] Sun Liang, Liang Shunlin, Yuan Wenping et al. Improving a Penman-Monteith evapotranspiration model by incorporating soil moisture control on soil evaporation in semi-arid area. International Journal of Digital Earth, 2013, 6: 134-156. DOI:10.1080/17538947.2013.783635.

\section{REFERENCES}

